\title{
V. Rybakov
}

\section{DISCRETE LINEAR TEMPORAL LOGIC WITH CURRENT TIME POINT CLUSTERS, DECIDING ALGORITHMS}

\author{
I wish to dedicate my paper to A. V. Kuznetsov - bright \\ logician and interesting, intelligent and creative man
}

\begin{abstract}
The paper* studies the logic $\mathcal{T} \mathcal{L}\left(\mathcal{N}_{C}^{\square_{w}^{+-}}\right)$- logic of discrete linear time with current time point clusters. Its language uses modalities $\diamond^{+}$ (possible in future) and $\diamond^{-}$(possible in past) and special temporal operations, $-\square_{w}^{+}$(weakly necessary in future) and $\square_{w}^{-}$(weakly necessary in past). We proceed by developing an algorithm recognizing theorems of $\mathcal{T} \mathcal{L}\left(\mathcal{N}_{C}^{\square_{w}^{+-}}\right)$, so we prove that $\mathcal{T} \mathcal{L}\left(\mathcal{N}_{C}^{\square_{w}^{+-}}\right)$is decidable. The algorithm is based on reduction of formulas to inference rules and converting the rules in special reduced normal form, and, then, on checking validity of such rules in models of singleexponential size in the rules. Also we consider the admissibility problem for $\mathcal{T} \mathcal{L}\left(\mathcal{N}_{C}^{\square}{ }_{w}^{+-}\right)$and show how to reduce the problem for admissibility to the decidability of $\mathcal{T} \mathcal{L}\left(\mathcal{N}_{C}^{\square_{w}^{+-}}\right)$itself using definable universal modalities.
\end{abstract}

Keywords: temporal logics, linear temporal logics, algorithms, Kripke structures, multi-modal logics, decidability, admissible inference rules.

${ }^{*}$ This research is supported by Engineering and Physical Sciences Research Council (EPSRC), U.K., grant EP/F014406/1. 


\section{Introduction}

Modal-type logics play an important role in various disciplines ranging from philosophy, through foundations of mathematics to applications in AI and CS. They provide excellent formalisms for studying the semantics of computation via graphs, e.g. transition systems, parse trees, Petri nets, decision diagrams, and flow charts. Most members of the AI community agree that logic has an important role to play in the central areas of AI research. This, in particular, follows from logical tools for deriving new knowledge (from known/accepted facts), which are based on logical consequence relation. Logical consequence, arguably the most fundamental concept in logic, is the relation that holds between a set of sentences (or propositions) and a sentence (proposition) when the latter follows from the former. Technique of non-classical logic (modal, multi-modal, temporal ones) has been involved to cover various aspects of problems for computerized modeling of intelligent behavior. Major applications to AI and CS was focused to reasoning about knowledge, time and computation (cf., for instance, Goldblatt [10, 11], van Benthem [31, 32], Gabbay, Hodkinson and Reynolds [5], Gabbay, Hodkinson [6]). Semantic tools for modal and temporal logics are often based on special sort of Kripke/Hinttikka models (special graphs), modal and temporal algebras (cf. Goldblatt [10, 11]).

It is relevant to say, that modal-like logics with linear alternative accessibility relations, linear temporal logics, were studied in details (and deserve it as most natural interpretation of the time flow). Say, Bull $[2,3]$ and Segerberg [30] found finite axiomatizations for many such logics, proved fmp (finite model property) (and hence decidability) for some these logics. Though [2] shows that temporal logic $\mathcal{L}_{Z}$ of integers with strict order does not have fmp. The decidability of $\mathcal{L}_{Z}$, first, it seems, was proved by D.Gabbay basing at Rabin's results of monadic 2nd order theories of successor functions (perhaps the decidability also followed from earlier research of D.Scott using automata). Litak and Wolter [19] showed that all finitely axiomatizable tense logics of linear time flows are decidable: coNPcomplete.

We are interested to extend the language of linear temporal logics by operations imitating possibility of discovery in time flow. The logic of discovery has a solid prehistory, maybe, starting from the monograph Logic of Discovery and Logic of Discourse by Jaakko Hintikka and Fernand Vandamme [18]. This logic has evident interdisciplinary direction and is influenced by various ideas coming from researchers with distinct background. 
One more direction merging pure proof theory and semantics is discovering admissible inference rules originated by Lorenzen [20]. Initially only particular interesting examples of admissible but not derivable rules were observed (cf. Harrop [14], Mints [21]). An affirmative solution for H.Friedman problem (1975) on existing an algorithm for solving admissibility problem for intuitionistic logic IPC was found in Rybakov [23]. Then S. Ghilardi [7] gave a solution of this problem via projective formulas, while P. Rozire [22] discovered the solution within proof theory. The area of study admissible inference rules was developed in a solid branch in non-classical logics (cf. Ghilardi [8, 9], Iemhoff [15], Jerábek [16], Rybakov [24]-[29]).

Next interest to our research came from the easy observation that linear temporal logics possess the definable universal modality. The universal modality, first was investigated in Goranko and Passy [13]. In study of hybrid logics, universal modality nowadays is one of standard constructors, cf. Acrces, Blackburn, Marx [1]. The idea to employ definable universal modalities for admissibility problem was looking rather attractive.

We address all this questions in this our paper, we study the logic $\mathcal{T L}\left(\mathcal{N}_{C}^{\square_{w}^{+-}}\right)$of linear discrete time with time point clusters and additional operations for discovery in time. The semantics for $\mathcal{T} \mathcal{L}\left(\mathcal{N}_{C}^{\square_{w}^{+-}}\right)$are Kripke/ Hintikka models generated by linear discrete time with time points being a clusters of states - uncertain situations. To study the properties of reasoning/computation modeled by these structures we introduce modal(temporal) like language including modalities $\diamond^{+}$(possible in future) and $\diamond^{-}$(possible in past) and else special temporal operations - weak necessities directed to future and past: $\square_{w}^{+}$(weakly necessary in future) and $\square_{w}^{-}$(weakly necessary in past). We discuss the language and rules for computing truth values of formulas of the suggested language in specified Kripke/Hintikka models, define formally the logic $\mathcal{T} \mathcal{L}\left(\mathcal{N}_{C}^{\square_{w}^{+-}}\right)$- the logic of linear time with weak necessity. The basic question we are dealing with first is construction of an algorithm recognizing theorems of $\mathcal{T} \mathcal{L}\left(\mathcal{N}_{C}^{\square_{w}^{+-}}\right)$and checking satisfiability of formulas. We propose an algorithm (so we show that $\mathcal{T} \mathcal{L}\left(\mathcal{N}_{C}^{\square_{w}^{+-}}\right.$) is decidable) based on reduction of formulas to rules and converting rules in special reduced normal form, and, then, on checking validity of such rules in models of single-exponential size in the rules. The technique which we use is borrowed from our research (cf. [23]-[29]) devoted to study inference rules in non-classical logics. At the second part of the paper, we show how to reduce in $\mathcal{T} \mathcal{L}\left(\mathcal{N}_{C}^{\square_{w}^{+-}}\right)$the admissibility problem for inference rules to the de- 
cidability of $\mathcal{T} \mathcal{L}\left(\mathcal{N}_{C}^{\square_{w}^{+-}}\right)$itself. It seems the logic $\mathcal{T} \mathcal{L}\left(\mathcal{N}_{C}^{\square_{w}^{+-}}\right)$has interesting new properties which been not considered yet in contemporary literature, and which are problematic to express by standard modal or temporal languages. The paper uses standard terminology and known facts about modal and temporal logic, though we supply the reader all necessary definitions and known facts to follow the paper.

\section{Notation, Preliminaries}

This paper explores special expansions of linear temporal logics; in general, temporal logics (in standard language) are, in own turn, special cases of bi-modal logics. Therefore we proceed by recalling definitions and notation concerning multi-modal logics. The language of multi-modal logics consists of the language of Boolean logic extended by new unary logical modal operations $\square_{i}$. Basically only finite amount of modal operations, $-\mathrm{n}-$, of $\square_{i}$, are used, then the introduced logics are called $n$-modal logic. Formation rules for formulas are standard.

A Kripke/Hintikka frame is a tuple $\mathcal{F}:=\left\langle F, R_{1}, \ldots, R_{n}\right\rangle$, where $F$ is the base of $\mathcal{F}$ - a non-empty set, and all $R_{i}$ are binary (accessibility) relations on $F .|\mathcal{F}|:=F, a \in \mathcal{F}$ is denotation for $a \in|\mathcal{F}|$. If, for a set of propositional letters $P$, a valuation $V$ of $P$ in $|\mathcal{F}|$ is defined, i.e. $V: P \rightarrow 2^{F}, \forall p \in P$ : $V(p) \subseteq F$, we call $\mathcal{M}:=\langle\mathcal{F}, V\rangle$ Kripke/Hintikka model (structure). The truth values of formulas are defined at elements of $\mathcal{F}$ by the following rules:

$$
\begin{aligned}
(\mathcal{F}, a) \Vdash_{V} p & \Longleftrightarrow a \in V(p), \forall p \in P, \forall a \in \mathcal{F} \\
(\mathcal{F}, a) \Vdash_{V} \varphi \wedge \psi & \Longleftrightarrow(\mathcal{F}, a) \Vdash_{V} \varphi \text { and }(\mathcal{F}, a) \Vdash_{V} \psi, \\
(\mathcal{F}, a) \Vdash_{V} \varphi \vee \psi & \Longleftrightarrow(\mathcal{F}, a) \Vdash_{V} \varphi \text { or }(\mathcal{F}, a) \Vdash_{V} \psi, \\
(\mathcal{F}, a) \Vdash_{V} \varphi \rightarrow \psi & \Longleftrightarrow(\mathcal{F}, a) \nVdash_{V} \varphi \text { or }(\mathcal{F}, a) \Vdash_{V} \psi, \\
(\mathcal{F}, a) \Vdash_{V} \neg \varphi & \Longleftrightarrow(\mathcal{F}, a) \nVdash_{V} \varphi, \\
(\mathcal{F}, a) \Vdash_{i} \varphi & \Longleftrightarrow \forall a \in \mathcal{F}\left(\left(a R_{i} b\right) \Rightarrow(\mathcal{F}, b) \Vdash \varphi\right) .
\end{aligned}
$$

Definition 1. For a Kripke-Hintikka structure $\mathcal{M}:=\langle\mathcal{F}, V\rangle$ and a formula $\varphi, \varphi$ is true in $\mathcal{M}$ (denotation: $\mathcal{M} \Vdash \varphi)$ if $\forall a \in \mathcal{F},(\mathcal{F}, a) \Vdash_{V} \varphi . \mathcal{F} \Vdash_{V} \varphi$ iff $\forall w \in \mathcal{F},(\mathcal{F}, w) \Vdash_{V} \varphi$.

Definition 2. For a class $\mathcal{K}$ of frames, the logic $\mathcal{L}(\mathcal{K})$ generated by $\mathcal{K}$ is the set of all formulas which are true in all models based on frames from $\mathcal{K}$.

For instance, for the basic minimal normal modal logic $K, K=\mathcal{L}\left(\mathcal{K}_{\text {fr }}\right)$, where $\mathcal{K}_{\text {fr }}$ is the set of all frames with single accessibility relation, for Lewis 
modal logic $S 4, S 4:=\mathcal{L}\left(\mathcal{K}_{\mathrm{rt}}\right)$, where $\mathcal{K}_{\mathrm{rt}}$ is the set of all frames with single reflexive and transitive accessibility relation. We can take in the definitions above only finite frames, i.e. $K$ and $S 4$ have so called finite model property (fmp). A semantic definition for temporal logics can be given based at special 2 -modal logics. Consider a set $\mathcal{K}$ of all 2 -frames of structure $\left\langle F, R, R^{-1}\right\rangle$, and the language with two modal operations $\square^{+}-$for $R$, and $\square^{-}-$for $R^{-1}$. The logic $\mathcal{L}(\mathcal{K})$ of $\mathcal{K}$ in this language is the temporal logic of $\mathcal{K}$. Operations $\diamond^{+}$ and $\diamond^{-}$are derivatives of $\square^{+}$and $\square^{-}$, as usual in modal logic. All Kripkecomplete temporal logics can be generated this way. For any logic $\mathcal{L}(\mathcal{K})$, a formula $\varphi$ is a theorem of $\mathcal{L}(\mathcal{K})$ if $\varphi \in \mathcal{L}(\mathcal{K}), \varphi$ is satisfiable in $\mathcal{K}$ if, for some valuation $V$ in some frame $\mathcal{F} \in \mathcal{K}, \varphi$ is true at an world of $\mathcal{F}$ w.r.t. $V$.

Linear Kripke complete temporal logics $\mathcal{L}(\mathcal{K})$ are based on classes $\mathcal{K}$ of frames with linear accessibility relation $R$ imitating time flaw. In this approach, any time point is just a state where propositions coded by formulas may be evaluated (computed).

We would like to extend this interpretation, looking at a time point as a model of current states in the given time point. What are these states? Maybe, the set of all human beings with their own knowledge and viewpoints, maybe the set of all Internet WEB sites and the set of all libraries and other information nodes available in a given time point, etc. So, based on this general idea, the semantics we consider are Kripke/Hintikka models based on linear graphs with circles described below. The frame is a graph $\mathcal{N}_{C}:=\left\langle\bigcup_{i \in N} C(i), R\right\rangle$, where $N$ is the set of all natural numbers, each $i \in N$ (any natural number $i$ ) is the time index for possible current events from now to future.

Any $C(i)$ is a finite set of all possible states in the time point $i$. In our formalism, any $C(i)$ is simply a set of elements (worlds in terms of Kripke/Hintikka semantics).

The time flow (say, imitating a computational process, an evolving of a system, a discovery search, a verification, just time flow from physical/philosophical viewpoint) is modeled by the binary accessibility relation $R$ in the frame $\mathcal{N}_{C}:=\left\langle\bigcup_{i \in N} C(i), R\right\rangle$, where for all elements $a$ and $b$ from $\bigcup_{i \in N} C(i)$,

$$
a R b \Longleftrightarrow a \in C(i) \text { and } b \in C(j) \text { and } i \leqslant j .
$$

$R$ imitates the discrete flow of time connecting states in time, so, $a R b$ means that $a$ and $b$ are some states at the same time point or the state $b$ may be achieved after the time point where the state $a$ will be accomplished. So, 
the flow of the time is supposed to be linear, which matches well with daily human experience. To reason about properties of the truth for statements in these frames, we introduce the following modal-like language

Take a set $P$ of propositional letters (to code facts, propositions, which may or may not happen, may be true or not true in the time flow of computation/reasoning). For each frame $\mathcal{N}_{C}$, we consider all valuations $V$ of $P$, which are mappings of $P$ into the set of all subsets of the set $\bigcup_{i \in N} C(i)$,

$$
\forall p \in P, \quad V(p) \subseteq \bigcup_{i \in N} C(i)
$$

If, for an element $a \in \bigcup_{i \in N} C(i)$, if $a \in V(p)$ we say the fact $\mathbf{p}$ holds in the state a. For example, consider the frame $\mathcal{N}_{C}:=\left\langle\bigcup_{i \in N}[2 i, 2 i+1], R\right\rangle$, with the valuation $V$ of the letter $p$, where $V(p):=\{2 i \mid i \in N\}$. Then the fact $p$ holds (is true) in a state of any cluster $[2 i, 2 i+1]$. This follows from the fact that any cluster $[2 i, 2 i+1]$ consists of an even number $2 i$, where $p$ is true, and followed by the odd number $2 i+1$. Let the frame $\mathcal{N}_{C}$ above to model the parallel computing, where at each computational step $i$ we achieve exactly two states $2 i$ and $2 i+1$ (numeration of which is not essential), but $2 i$ always is the state, where the property $p$ - to have consistent outputs-holds. Then the frame $\mathcal{N}_{C}$ models the special parallel computing, where, at each computational step, at least one state with consistent outputs is discoverable. To construct formulas, we use all Boolean logical operations: $\wedge$ (and), $\vee$ (or), $\neg$ (not), $\rightarrow$ (implease), and extend them by the modal operations $\diamond^{+}$ (possible in future), $\diamond^{-}$(possible in past), $\square_{w}^{+}$(weak necessary in future) and $\square_{w}^{-}$(weak necessary in past). The formation rules for formulas (wffs) are the standard ones:

(i) Any propositional letter from $P$ is a well formed formula (wff).

(ii) If $\varphi$ and $\psi$ are wffs then $\varphi \wedge \psi, \varphi \vee \psi, \varphi \rightarrow \psi$ and $\neg \varphi$ are also wffs.

(iii) If $\varphi$ is a wff then $, \diamond^{+} \varphi, \diamond^{-} \varphi, \square^{+} \varphi$ and $\square^{-} \varphi$ are also wffs.

The rules for computing of truth values for formulas in models $\mathcal{N}_{C}$ with valuations $V$ of propositions $P$ are as follows. In the notation below $\left(\mathcal{N}_{C}, a\right) \Vdash_{V} \varphi$ is meant to say the formula $\varphi$ in true at the state $a$ in the model $\mathcal{N}_{C}$ w.r.t. valuation $V . \forall p \in P, \forall a \in \mathcal{N}_{C}:\left(\mathcal{N}_{C}, a\right) \Vdash_{V} p \Longleftrightarrow a \in V(p)$. Steps for Boolean operations are standard, as described above for multimodal logics. The steps for $\diamond^{+}$and $\diamond^{-}$are also standard, as temporal 
operation for future and past:

$$
\begin{aligned}
& \left(\mathcal{N}_{C}, a\right) \Vdash_{V} \diamond^{+} \varphi \Longleftrightarrow \exists b \in \mathcal{N}_{C}\left[a R b \text { and }\left(\mathcal{N}_{C}, b\right) \Vdash_{V} \varphi\right], \\
& \left(\mathcal{N}_{C}, a\right) \Vdash_{V} \diamond^{-} \varphi \Longleftrightarrow \exists b \in \mathcal{N}_{C}\left[b R a \text { and }\left(\mathcal{N}_{C}, b\right) \Vdash_{V} \varphi\right] .
\end{aligned}
$$

But we interpret $\square_{w}^{+}$and $\square_{w}^{-}$as not in standard modal or temporal operations:

$$
\begin{aligned}
& \left(\mathcal{N}_{C}, a\right) \Vdash_{V} \square_{w}^{+} \varphi \Longleftrightarrow\left[a \in C(i) \Rightarrow\left[\forall j \geqslant i \exists b \in C(j)\left(\mathcal{N}_{C}, b\right) \Vdash_{V} \varphi\right]\right], \\
& \left(\mathcal{N}_{C}, a\right) \Vdash_{V} \square_{w}^{-} \varphi \Longleftrightarrow\left[a \in C(i) \Rightarrow\left[\forall j \leqslant i \exists b \in C(j)\left(\mathcal{N}_{C}, b\right) \Vdash_{V} \varphi\right]\right] .
\end{aligned}
$$

So, computation of the operations $\square_{w}^{+}$and $\square_{w}^{-}$are nonstandard. $\square_{w}^{+} \varphi$ says that $\varphi$ is weakly necessary in future - in any time cluster in future, there is a state where $\varphi$ holds, while the dual one $-\square_{w}^{-} \varphi$ says that $\varphi$ is weakly necessary in past - for any time cluster in past, there is a state where $\varphi$ is true. Thus, we suggest a thinner treatment of the operation $\square$ as a variant of week necessity. This specification of $\square_{w}$ makes the logic we offer to be different from all standard temporal or modal logic - being a non-classical temporal logic.

Definition 3. The logic $\mathcal{T} \mathcal{L}\left(\mathcal{N}_{C}^{\square_{w}^{+-}}\right)$is the set of all formulas which are true in any state of any frame $\mathcal{N}_{C}$ w.r.t. any valuation.

We enumerate below some standard modal laws which being referred to $\square_{w}^{+}$do not hold in $\mathcal{T} \mathcal{L}\left(\mathcal{N}_{C}^{\square}\right)$.

Lemma 4. The following holds $\square_{w}^{\circ} p \rightarrow p \notin \mathcal{T} \mathcal{L}\left(\mathcal{N}_{C}^{\square_{w}^{+-}}\right), \square_{w}^{\circ} p \equiv \neg \diamond^{\circ} \neg p \notin$ $\mathcal{T} \mathcal{L}\left(\mathcal{N}_{C}^{\square_{w}^{+-}}\right)$and $\diamond^{\circ} p \equiv \neg \square_{w}^{\circ} \neg p \notin \mathcal{T} \mathcal{L}\left(\mathcal{N}_{C}^{\square_{w}^{+-}}\right), \square_{w}^{\circ}(p \rightarrow q) \rightarrow\left(\square_{w}^{\circ} p \rightarrow \square_{w}^{\circ} q\right) \notin$ $\mathcal{T} \mathcal{L}\left(\mathcal{N}_{C}^{\square} \square_{w}^{+-}\right)$, where $\circ \in\{+,-\}$.

The proof is easy computation. So, $\square_{w}^{+}$-fragment of $\mathcal{T} \mathcal{L}\left(\mathcal{N}_{C}^{\square_{w}^{+-}}\right)$is not a fragment of any modal logic, $\square_{w}^{+}$is not a modal or temporal operation in standard understanding. And evidently, $\square_{w}^{\circ}$ and $\diamond^{\circ}$ are no longer mutual counterparts of each other, so $\mathcal{T} \mathcal{L}\left(\mathcal{N}_{C}^{\square_{w}^{+-}}\right)$differs with any standard normal or not-normal modal/temporal logic (because $\square_{w}^{\circ} p \equiv \neg \diamond^{\circ} \neg p$ and $\diamond^{\circ} p \equiv$ $\neg \square_{w}^{\circ} \neg p$ do not hold in $\left.\mathcal{T} \mathcal{L}\left(\mathcal{N}_{C}^{\square_{w}^{+-}}\right)\right)$. So, the logic is indeed a bit unusual. It is interesting whether $\square_{w}^{\circ}$ and $\diamond^{\circ}$ may be mutually expressed by some other ways. At the same time, it is immediate to show that 
Lemma 5. The following holds

$$
\begin{aligned}
& \square_{w}^{\circ} p \rightarrow \square_{w}^{\circ} \square_{w}^{\circ} p \in \mathcal{T} \mathcal{L}\left(\mathcal{N}_{C}^{\square_{w}^{+-}}\right), \\
& \square_{w}^{\circ}\left(\square_{w}^{\circ} p \rightarrow \square_{w}^{\circ} q\right) \vee \square_{w}^{\circ}\left(\square_{w}^{\circ} q \rightarrow \square_{w}^{\circ} p\right) \in \mathcal{T} \mathcal{L}\left(\mathcal{N}_{C}^{\square_{w}^{+-}}\right), \\
& \varphi \in \mathcal{T} \mathcal{L}\left(\mathcal{N}_{C}^{\square_{w}^{+-}}\right) \Rightarrow \square_{w}^{\circ} \varphi \in \mathcal{T} \mathcal{L}\left(\mathcal{N}_{C}^{\square_{w}^{+-}}\right) .
\end{aligned}
$$

Expressive power of $\mathcal{T} \mathcal{L}\left(\mathcal{N}_{C}^{\square_{w}^{+-}}\right)$is visible when we combine both modalities $\square_{w}^{\circ}$ and $\diamond^{\circ}$ in formulas, the language became to be much more flexible and expressible. For instance, the truth of formula $\diamond^{+} \square_{w}^{+} \varphi$ in a frame $\mathcal{N}_{C}$ w.r.t a valuation (labeling) $V$ means that a time point $i$ is possible, where, since $i$ in any time cluster the property $\varphi$ is discoverable. The truth of the formula $\square_{w}^{+} p \wedge \square_{w}^{+} \neg p \rightarrow \neg \diamond^{+} q$ would mean that if, in any time point, both $p$ and $\neg p$ are discoverable then in any length of time flow (a computation) $q$ cannot be discovered. All such properties are difficult to be expressed in the standard interpretation of modal or temporal language.

We will study properties of $\mathcal{T} \mathcal{L}\left(\mathcal{N}_{C}^{\square^{+-}}\right)$in order to know which logical laws hold for it, say, to know whether two formulas $\varphi$ and $\psi$ are equivalent, i.e. whether $\varphi \equiv \psi \in \mathcal{T} \mathcal{L}\left(\mathcal{N}_{C}^{\square_{w}^{+-}}\right)$, whether a formula $\varphi$ is a theorem (a logical law for $\left.\mathcal{T} \mathcal{L}\left(\mathcal{N}_{C}^{\square_{w}^{+-}}\right)\right)$, i.e. whether $\varphi \in \mathcal{T} \mathcal{L}\left(\mathcal{N}_{C}^{\square_{w}^{+-}}\right)$.

Definition 6. A logic $\mathcal{L}$ is said to be decidable if there is an algorithms, which, for any formula $\varphi$, answer whether $\varphi \in \mathcal{L}$ (i.e. whether $\varphi$ is a theorem of $\mathcal{L})$.

The problem of decidability is one of basic problems for any logic. For instance, the satisfiability problem may be reduced to decidability. Indeed, a formula $\varphi$ is satisfiable iff $\neg \varphi$ is not a theorem. We address the decidability problem to $\mathcal{T} \mathcal{L}\left(\mathcal{N}_{C}^{\square_{w}^{+-}}\right)$in the following section.

\section{Results, Decidability}

The prime question we are dealing with in this section is how, for any given formula $\varphi$, to determine whether $\varphi$ is a theorem of $\mathcal{T} \mathcal{L}\left(\mathcal{N}_{C}^{\square_{w}^{+-}}\right)$, whether $\varphi \in \mathcal{T} \mathcal{L}\left(\mathcal{N}_{C}^{\square_{w}^{+-}}\right)$. Actually $\mathcal{T} \mathcal{L}\left(\mathcal{N}_{C}^{\square_{w}^{+-}}\right)$is an expansion of bimodal logic, and we can borrow some evolved technique to approach this task. Several ways are possible and we apply a technique based on our own approach tested for several logics (cf. [23]-[29]). This approach uses a representation of formulas by rules, and converting rules in a special normal reduced form. Recall, a 
(sequential) rule is an expression

$$
\mathbf{r}:=\frac{\varphi_{1}\left(x_{1}, \ldots, x_{n}\right), \ldots, \varphi_{m}\left(x_{1}, \ldots, x_{n}\right)}{\psi\left(x_{1}, \ldots, x_{n}\right)},
$$

where $\varphi_{1}\left(x_{1}, \ldots, x_{n}\right), \ldots, \varphi_{m}\left(x_{1}, \ldots, x_{n}\right)$ and $\psi\left(x_{1}, \ldots, x_{n}\right)$ are some formulas constructed out of letters $x_{1}, \ldots, x_{n}$. Letters $x_{1}, \ldots, x_{n}$ are called variables of $\mathbf{r}$, and are denoted by $\operatorname{Var}(r)$. A formula $\varphi$ is valid in a frame $\mathcal{N}_{C}$ (notation: $\mathcal{N}_{C} \Vdash \varphi$ ) if, for any valuation $V$ of $\operatorname{Var}(\varphi)$ and, for any element $a$ of $\mathcal{N}_{C},\left(\mathcal{N}_{C}, a\right) \Vdash_{V} \varphi$.

Definition 7. A rule $\mathbf{r}$ is said to be valid in the Kripke model $\left\langle\mathcal{N}_{C}, V\right\rangle$ with the valuation $V$ (we will use notation $\mathcal{N}_{C} \Vdash_{V} \mathbf{r}$ ) if

$$
\forall a\left(\left(\mathcal{N}_{C}, a\right) \Vdash_{V} \bigwedge_{1 \leqslant i \leq m} \varphi_{i}\right) \Rightarrow \forall a\left(\mathcal{N}_{C}, a\right) \Vdash_{V} \psi
$$

Otherwise we say $\mathbf{r}$ is refuted in $\mathcal{N}_{C}$, or refuted in $\mathcal{N}_{C}$ by $V$, and write $\mathcal{N}_{C} \nVdash_{V} \mathbf{r}$.

A rule $\mathbf{r}$ is valid in a frame $\mathcal{N}_{C}$ (notation: $\mathcal{N}_{C} \Vdash \mathbf{r}$ ) if, for any valuation $V$ of $\operatorname{Var}(\mathbf{r}), \mathcal{N}_{C} \Vdash_{V} \mathbf{r}$. A rule $\mathbf{r}$ is said to have the reduced normal form if $\mathbf{r}=\varepsilon_{c} / x_{1}$ where

$$
\begin{aligned}
\varepsilon_{c}:= & \bigvee_{1 \leqslant j \leq m}\left(\bigwedge _ { 1 \leqslant i \leqslant n } \left[x_{i}^{t(j, i, 0)} \wedge\left(\square_{w}^{+} x_{i}\right)^{t(j, i, 1)} \wedge\left(\diamond^{+} x_{i}\right)^{t(j, i, 2)} \wedge\right.\right. \\
& \left.\left.\wedge\left(\square_{w}^{-} x_{i}\right)^{t(j, i, 3)} \wedge\left(\diamond^{-} x_{i}\right)^{t(j, i, 4)}\right]\right),
\end{aligned}
$$

and $x_{s}$ are certain letters (variables), $t(j, i, z) \in\{0,1\}$ and, for any formula $\alpha$ above, $\alpha^{0}:=\alpha, \alpha^{1}:=\neg \alpha$. For any formula $\varphi$ we can convert it in the rule $x \rightarrow x / \varphi$ and employ technique of reduced normal forms as follows.

Definition 8. Given a rule $\mathbf{r}_{\mathbf{n f}}$ in the reduced normal form, $\mathbf{r}_{\mathbf{n f}}$ is said to be a normal reduced form for a rule $\mathbf{r}$ iff, for any frame $\mathcal{N}_{C}, \mathcal{N}_{C} \Vdash \mathbf{r} \Longleftrightarrow$ $\mathcal{N}_{C} \Vdash \mathbf{r}_{\text {nf }}$.

Based on proofs of Lemma 3.1.3 and Theorem 3.1.11 from [24] directed to modal inference rules, by similar technique, we can obtain

Theorem 9. There exists an algorithm running in (single) exponential time, which, for any given rule $\mathbf{r}$, constructs its normal reduced form $\mathbf{r}_{\mathbf{n f}}$. 
Proof. We just sketch the proof below because the construction is simple. Given a rule $\mathbf{r}=\varphi_{1}\left(x_{1}, \ldots, x_{n}\right), \ldots, \varphi_{m}\left(x_{1}, \ldots, x_{n}\right) / \psi\left(x_{1}, \ldots, x_{n}\right) . \mathbf{r}$ is equivalent to

$$
\mathbf{r}_{0}=\varphi_{1}\left(x_{1}, \ldots, x_{n}\right) \wedge \cdots \wedge \varphi_{m}\left(x_{1}, \ldots, x_{n}\right) \wedge x_{c} \equiv \psi\left(x_{1}, \ldots, x_{n}\right) / x_{c}
$$

where $x_{c}$ is a new variable. Therefore we can restrict the case by consideration only rules in the form $\mathbf{c}=\varphi\left(x_{1}, \ldots, x_{n}\right) / x_{c}$.

If $\varphi=\alpha \circ \beta$, where $\circ$ is a binary logical operation and both formulas $\alpha$ and $\beta$ are not simply variables or unary logical operations applied to variables (which both we call final formulas), take two new variables $x_{\alpha}$ and $x_{\beta}$ and the rule

$$
\mathbf{r}_{1}:=x_{\alpha} \circ x_{\beta} \wedge x_{\alpha} \equiv \alpha \wedge x_{\beta} \equiv \beta / x_{c} .
$$

If one from formulas $\alpha$ or $\beta$ is final and another one not, we apply this transformation to only non-final formula. It is clear that $\mathbf{r}$ and $\mathbf{r}_{\mathbf{1}}$ are equivalent w.r.t. validity in frames.

If $\varphi=* \alpha$, where $*$ is a unary logical operation and $\alpha$ is not a variable, take a new variable $x_{\alpha}$ and the rule $\mathbf{r}_{1}:=* x_{\alpha} \wedge x_{\alpha} \equiv \alpha / x_{c}$. Again $\mathbf{r}$ and $\mathbf{r}_{\mathbf{1}}$ are equivalent.

We continue this (similar) transformation over the resulting rules

$$
\frac{\bigwedge_{j \in J_{1}} \gamma_{j} \wedge \bigwedge_{i \in I_{1}} x_{\alpha_{i}} \equiv \alpha_{i}}{x_{c}}
$$

until all formulas $\alpha_{i}$ and $\gamma_{j}$ in the premise of the resulting rules will be either atomic formulas - logical operations applied to variables - or variables. Evidently this transformation is polynomial. Next, we transform the premise of the resulting rule in the disjunctive normal form and make disjunctive normal form to be perfect (having the disjunctive members to be uniform length and containing all the components required in the definition of reduced normal forms) and obtain as the result an equivalent consecution $\mathbf{r}_{\mathbf{2}}$. This transformation, as well as all known ones for reduction Boolean formulas to disjunctive normal forms, is exponential. But then $\mathbf{r}$ has the required form.

To discuss this transformation more, in (Rybakov [24]), as an immediate consequence of the reduction of modal rules to normal forms (as above), it was shown (Corollary 3.1.27) that any normal modal logic extending $K 4$ can be axiomatized by formulas of modal degree at most 2 (known result, 
Zakharyaschev, 1992). It can be done with impressive ease. Take a modal formula $\alpha$, its validity is equivalent to the validity of the rule $r:=\top / \alpha$. Take its reduced normal form $r f(r)=\beta / x_{1}$. For any rule $r_{1}=\gamma / \delta$, its transformation to the semi-universal formula $f\left(r_{1}\right)$ is the formula $\square \gamma \wedge \gamma \rightarrow \delta$. And, for any modal logic $L$ over $K 4, L \oplus \alpha=L \oplus f(r f(r))$, so all works. In the case of temporal logics, using the transformation of rules to the reduced normal forms described above, we can obtain a similar result: any temporal logic (it is unimportant transitive or not) enriched with the universal modality can be axiomatized by formulas of modal/temporal degree at most 2 . The same holds for simply temporal logics where $\square^{+}$and $\square^{-}$are $K 4$-modalities.

It is immediate to see that a formula $\varphi$ is valid in a frame $\mathcal{N}_{C}$ iff the rule $x \rightarrow x / \varphi$ is valid in $\mathcal{N}_{C}$, so from Theorem 9 we obtain

LEMma 10. A formula $\varphi$ is a theorem of $\mathcal{T} \mathcal{L}\left(\mathcal{N}_{C}^{\square}{ }_{w}^{+-}\right)$iff the rule $(x \rightarrow x / \varphi)_{\mathrm{nf}}$ is valid in any frame $\mathcal{N}_{C}$.

Thus, to solve the question about decidability of $\mathcal{T L}\left(\mathcal{N}_{C}^{\square_{w}^{+-}}\right)$it is sufficient to find an algorithm recognizing rules in reduced normal form which are valid in all frames $\mathcal{N}_{C}$. To describe our algorithm we need the following special finite Kripke models. Take any frame $\mathcal{N}_{C}$ and some numbers $n, m$, where $m>n>1$. The frame $\mathcal{N}_{C}(n, m)$ has the following structure:

$$
\mathcal{N}_{C}(n, m):=\left\langle\bigcup_{1 \leqslant i \leqslant m} C(i), R\right\rangle,
$$

where $R$ is the accessibility relation from $\mathcal{N}_{C}$ extended by pairs $(x, y)$, where $x \in C\left(j_{1}\right), y \in C\left(j_{2}\right)$ and $j_{1}, j_{2} \in[n, m]$, so $x R y$ holds for all such pairs. If given a valuation $V$ of letters from a formula $\varphi$ in $\mathcal{N}_{C}(n, m)$, the truth values of $\varphi$ can be defined at elements of $\mathcal{N}_{C}(n, m)$ by the same rules as for frames $\mathcal{N}_{C}$ above (simply in accordance with standard computing of truth values for modal/temporal operations). Just in case, we describe below steps for operations $\diamond^{+}, \square_{w}^{+}, \diamond^{-}$and $\square_{w}^{-}$:

$$
\begin{aligned}
& \left(\mathcal{N}_{C}(n, m), a\right) \Vdash_{V} \diamond^{+} \varphi \Longleftrightarrow \exists b \in \mathcal{N}_{C}\left[a R b \text { and }\left(\mathcal{N}_{C}(n, m), b\right) \Vdash_{V} \varphi\right], \\
& \left(\mathcal{N}_{C}(n, m), a\right) \Vdash_{V} \square_{w}^{+} \varphi \Longleftrightarrow[(a \in C(i)) \&(i \leqslant n) \& \\
& \left.\left(\forall j(m \geqslant j \geqslant i) \exists b \in C(j)\left(\mathcal{N}_{C}(n, m), b\right) \Vdash_{V} \varphi\right)\right] \text { or } \\
& {\left[(a \in C(i)) \&(i>n) \&\left(\forall j(n \leqslant j \leqslant m) \exists c \in C(j)\left(\mathcal{N}_{C}(n, m), b\right) \Vdash_{V} \varphi\right)\right],} \\
& \left(\mathcal{N}_{C}(n, m), a\right) \Vdash_{V} \diamond^{-} \varphi \Longleftrightarrow \exists b \in \mathcal{N}_{C}\left[b R a \text { and }\left(\mathcal{N}_{C}(n, m), b\right) \Vdash_{V} \varphi\right],
\end{aligned}
$$




$$
\begin{aligned}
\left(\mathcal{N}_{C}(n, m), a\right) \Vdash_{V} \square_{w}^{-} \varphi \Longleftrightarrow[(a \in C(i)) \&(i \leqslant n) \& \\
\left.\left(\forall j(j \leqslant i) \exists b \in C(j)\left(\mathcal{N}_{C}(n, m), b\right) \Vdash_{V} \varphi\right)\right] \text { or } \\
\\
{\left[(a \in C(i)) \&(i>n) \&\left(\forall j \exists c \in C(j)\left(\mathcal{N}_{C}(n, m), c\right) \Vdash_{V} \varphi\right)\right] . }
\end{aligned}
$$

Notice that the interpretations for $\square_{w}^{+}$and $\square_{w}^{-}$are not mutually dual. Using this modified Kripke structures $\mathcal{N}_{C}(n, m)$ we derive

Lemma 11. A rule $\mathbf{r}_{\mathbf{n f}}$ in the reduced normal form is refuted in a frame $\mathcal{N}_{C}$ w.r.t. a valuation $V$ if and only if $\mathbf{r}_{\mathbf{n f}}$ is refuted in a frame $\mathcal{N}_{C}(n, m)$ by a valuation $V_{1}$, where

- The size of any cluster $C(i)$ in $\mathcal{N}_{C}(n, m)$ is linear in $\mathbf{r}_{\mathbf{n f}}$.

- $n$ and $m$ are single-exponential in $\mathbf{r}_{\mathbf{n f}}$.

- For any $C(i) \subset \mathcal{N}_{C}(n, m)$ with $i \geqslant n$, there is a $C(j) \subset \mathcal{N}_{C}(n, m)$ with $j<$ $n$ such that $C(j)$ and $C(i)$ are isomorphic as models w.r.t. the valuation $V_{1}$.

Proof. Let $\mathcal{N}_{C}:=\left\langle\bigcup_{i \in N} C(i), R\right\rangle$ and $\mathcal{N}_{C} \nVdash_{V} \mathbf{r}_{\text {nf }}$. Let $\operatorname{Pr}\left(r_{\mathrm{nf}}\right)$ be the premise of $\mathbf{r}_{\mathbf{n f}}, D\left(r_{\mathrm{nf}}\right)$ be the set of all disjuncts from $\operatorname{Pr}\left(r_{\mathrm{nf}}\right)$ and $\operatorname{Var}\left(r_{\mathrm{nf}}\right)$ be the set of all letters from $\mathbf{r}_{\mathbf{n f}}$. For any $D \in D\left(r_{n f}\right), \operatorname{Sub}(D)$ is the set of all subformulas of $D$. By $\mathcal{N}_{C} \nVdash_{V} \mathbf{r}_{\mathbf{n f}}$, for any $a \in \mathcal{N}_{C}$, there is a unique disjunct $D$ from $D\left(r_{\text {nf }}\right)$ such that $\left(\mathcal{N}_{C}, a\right) \Vdash_{V} D$. In the sequel we will denote this unique disjunct by $D_{\mathcal{N}_{C}}(a)$. First, instead of doing standard filtration on clusters $C(i)$, we will restrict the size of clusters $C(i)$ by simply rarefication. In any $C(i)$ we choose only one world $a$ from $\left\{b \mid b \in C(i),\left(\mathcal{N}_{C}, b\right) \Vdash_{V}\right.$ $\left.D_{\mathcal{N}_{C}}(a)\right\}$. This reducing the number of worlds in clusters evidently does not change the truth values of formulas from $D\left(r_{\mathrm{nf}}\right)$, so the resulting model will also invalidate $\mathbf{r}_{\mathbf{n f}}$ by $V$. So, we can admit that the size of clusters in $\mathcal{N}_{C}$ is linear in $\mathbf{r}_{\mathbf{n f}}$. In the sequel we consider any cluster $C(i)$ as the Kripke model with the induced valuation $V$. In the sequel we will write $C(i)=C(j)$ if $C(i)$ and $C(j)$ are isomorphic as models. By our rarefication, the set of all such $C(i)$ (as Kripke models w.r.t. $V$ ) is at most exponential in $\mathbf{r}_{\mathbf{n f}}$.

By $\mathcal{N}_{C} \nVdash_{V} \mathbf{r}_{\mathbf{n f}}$ it follows $\left(\mathcal{N}_{C}, a_{0}\right) \Vdash_{V} \neg x_{1}$ for $a_{0} \in C\left(i_{0}\right)$ and some $i_{0}$, fix a such minimal $i_{0}$. Consider minimal number $k_{0}>i_{0}$ such that

$$
\left\{C(j) \mid j<k_{0}\right\} \supseteq\{C(j) \mid j \in N\} .
$$

Recall we consider here and in what follows all $C(j)$ as Kripke models w.r.t. $V$. Take minimal number $i_{\max }>k_{0}+1$, where

$$
\forall i \in N,\{C(j) \mid j \leqslant i\} \subseteq\left\{C(j) \mid j \leqslant i_{\max }\right\} .
$$


Next, we take the minimal number $i_{\max }^{\min } \geqslant i_{\max }+2$ such that

$$
\forall i \in N,\left\{C(j) \mid j \geqslant i>i_{\max }\right\} \supseteq\left\{C(j) \mid j \geqslant i_{\max }^{\min }\right\} .
$$

For any $C(i) \in\left\{C(j) \mid j \geqslant i_{\max }^{\min }\right\}$ choose the minimal $j_{C(i)}>i_{\max }^{\min }+1$ where $C\left(j_{C(i)}\right)=C(i)$. Among all such $j_{C(i)}$ choose the maximal, one $-j_{0}$.

Let $n:=i_{\max }^{\min }$ and $m:=j_{0}+1$. Consider the model $\mathcal{N}_{C}(n, m)$ obtained from $\mathcal{N}_{C}$ as described above with the induced from $\mathcal{N}_{C}$ valuation $V$.

To continue the proof we need the following lemma:

Lemma 12. For any $a \in \mathcal{N}_{C}(n, m),\left(\mathcal{N}_{C}(n, m), a\right) \Vdash_{V} D_{\mathcal{N}_{C}}(a)$.

Proof. It follows by simple conventional computation using (2), (3) and the choice of $j_{0}$, and therefore detailed proof is left to the reader.

Numbers $n$ and $m$ are not bounded yet. But we can reduce the number of clusters above $C(n)$ as follows. Let $S:=\{C(i) \mid i \in[n, m]\}$. For any $C(i) \in S$ choose a single $C\left(i_{1}\right)$ in $\{C(j) \mid j \in[n, m], C(j)=C(i)\}$. Modify $\mathcal{N}_{C}(n, m)$ as follows

$$
\mathcal{N}_{C}(n):=\left\langle\bigcup_{i \in[1, n]} C(i) \cup \bigcup_{i \in[n+1, m]} C\left(i_{1}\right), R, V\right\rangle,
$$

where $R$ and $V$ are transferred from $\mathcal{N}_{C}(n, m)$.

Lemma 13. For any $a \in \mathcal{N}_{C}(n),\left(\mathcal{N}_{C}(n), a\right) \Vdash_{V} D_{\mathcal{N}_{C}}(a)$.

Proof. follows from Lemma 12 and the choice of clusters $C\left(i_{1}\right)$ above by simple computation and is left to the reader.

Note that the number of clusters $C\left(i_{1}\right)$ is at most exponential in $\mathbf{r}_{\mathbf{n f}}$. So, we can assume that $\mathbf{r}_{\mathbf{n f}}$ is invalidated by a valuation $V$ in a model $\mathcal{N}_{C}(n, m)$ with clusters $C(i)$ linear in $\mathbf{r}_{\mathbf{n f}}$ and where $m-n$ is at most exponential in $\mathbf{r}_{\mathbf{n f}}$. It only remains to bound $n$. For this, for any $C(i)$ where $i \leqslant n-1$, consider the set

$$
\text { Clones }(C(i)):=\{C(j) \mid j \leqslant n-1, C(j)=C(i)\} .
$$

Let

$$
\begin{aligned}
C(i, \min ) & :=C\left(j_{1}\right), \quad j_{1}=\min \{j \mid C(j) \in \operatorname{Clones}(C(i)), \\
C(i, \max ) & :=C\left(j_{2}\right), \quad j_{2}=\max \{j \mid C(j) \in \operatorname{Clones}(C(i)) .
\end{aligned}
$$


Modify now our model $\mathcal{N}_{C}(n, m)$ with the valuation $V$ as follows:

$$
\mathcal{N}_{C}(n, m, 1):=\left\langle\bigcup_{i \in[1, n-1]}[C(i, \min ) \cup C(i, \max )] \cup \bigcup_{i \in[n, m]} C(i), R, V\right\rangle,
$$

where $R$ and $V$ are transferred from $\mathcal{N}_{C}(n, m)$.

Lemma 14. For any $a \in \mathcal{N}_{C}(n, m, 1),\left(\mathcal{N}_{C}(n, m, 1), a\right) \Vdash_{V} D_{\mathcal{N}_{C}}(a)$.

Proof. Follows from the construction of the model $\mathcal{N}_{C}(n, m, 1)$ above and the choice of clusters $C(i, \min )$ and $C(i, \max )$ by simple routine verification which is left to the reader.

So, $\mathcal{N}_{C}(n, m, 1)$ invalidates $\mathbf{r}_{\mathbf{n f}}$ by $V$, and $\mathcal{N}_{C}(n, m, 1)$ has form $\mathcal{N}_{C}\left(n_{1}, m_{1}\right)$, where $n_{1}$ and $m_{1}$ are at most exponential in $\mathbf{r}_{\mathbf{n f}}$, clusters are linear in $\mathbf{r}_{\mathbf{n f}}$ and (iii) from Lemma 11 holds for this $\mathcal{N}_{C}\left(n_{1}, m_{1}\right)$ by (1), (2), (3) and the choice of $j_{0}$. Thus, we proved the necessity in Lemma 11.

Sufficiency. Vise versa, assume $\mathbf{r}_{\mathbf{n f}}$ is invalidated by a valuation $V_{1}$ in some $\mathcal{N}_{C}(n, m)$ satisfying conditions of Lemma 11 . We transform $\mathcal{N}_{C}(n, m)$ by standard bulldozing technique. We roll the whole interval of all clusters $[C(n), C(n+1), \ldots, C(m-1), C(m)]$ towards 'future' transferring the valuation $V_{1}$ as identical on copies. The resulting Kripke model has form $\mathcal{N}_{C}$. Let, for any cluster $C(i)$ from $\mathcal{N}_{C}, f^{-1} C(i)$ be the pre-image of $C(i)$ in $\mathcal{N}_{C}(n, m)$; note that $f^{-1} C(i)$ and $C(i)$ are identical as Kripke models. Let, for any $a \in C(i) \subset \mathcal{N}_{C}, f^{-1}(a)$ to be pre-image of $a$ in $\mathcal{N}(n, m)$.

Lemma 15. For any $a \in \mathcal{N}_{C},\left(\mathcal{N}_{C}, a\right) \Vdash_{V} D_{\mathcal{N}_{C}(n, m}\left(f^{-1}(a)\right)$.

Proof. follows from (iii) in Lemma 11 by standard routine verification.

Therefore $\mathcal{N}_{C}$ refutes the rule $\mathbf{r}_{\mathbf{n f}}$ as well as $\mathcal{N}_{C}(n, m)$ does. This concludes the proof of Lemma 11.

Combining Theorem 9 and lemmas 10 and 11 we derive

ThEOREM 16. The logic $\mathcal{T} \mathcal{L}\left(\mathcal{N}_{C}^{\square_{w}^{+-}}\right)$is decidable. The algorithm of verification formulas to be a theorems consists of verification validity of rules in reduced normal form in Kripke frames of single-exponential size in the rules.

The overall complexity of the algorithm includes as well the reduction of rules to normal reduced forms, but this complexity is single exponential (the same as the complexity of reduction any Boolean formula to the disjunctive normal form). This theorem is a departure point for study admissible in $\mathcal{T} \mathcal{L}\left(\mathcal{N}_{C}^{\square_{w}^{+-}}\right)$inference rules, which is undertaken in the next section. 


\section{Reduction Admissible in $\mathcal{T L}\left(\mathcal{N}_{C}^{\square}{ }^{+-}\right)$Rules to Theorems of $\mathcal{T L}\left(\mathcal{N}_{C}^{\square_{w}^{+-}}\right)$}

To recall definitions, given a $\operatorname{logic} \mathcal{L}, \operatorname{Form}_{\mathcal{L}}$ is the set of all formulas in the language of $\mathcal{L}$.

Definition 17. A rule

$$
\mathbf{r}:=\frac{\varphi\left(x_{1}, \ldots, x_{n}\right), \ldots, \varphi_{m}\left(x_{1}, \ldots, x_{n}\right)}{\psi\left(x_{1}, \ldots, x_{n}\right)},
$$

is said to be admissible for a logic $\mathcal{L}$ if, $\forall \alpha_{1} \in \operatorname{Form}_{\mathcal{L}}, \ldots, \forall \alpha_{n} \in \operatorname{Form}_{\mathcal{L}}$,

$$
\left[\bigwedge_{1 \leqslant i \leqslant m} \varphi_{i}\left(\alpha_{1}, \ldots, \alpha_{n}\right) \in \mathcal{L}\right] \Longrightarrow \psi\left(\alpha_{1}, \ldots, \alpha_{n}\right) \in \mathcal{L} .
$$

Thus, for any admissible rule, any instance into the premises converting all of them into theorems of $\mathcal{L}$ also turns the conclusion to be a theorem. For instance, the Lemmon-Scott rule

$$
\frac{\square(\square(\square \diamond \square p \rightarrow \square p) \rightarrow(\square p \vee \square \neg \square p))}{\square \diamond \square p \vee \square \neg \square p}
$$

is admissible but not derivable in standard Hilbert-style axiomatizations for modal logics S4, S4.1 and Grz (cf. [24]).

We need one more useful notion from multi-modal logics to develop our technique. For any frame $\mathcal{F}:=\left\langle F, R_{1}, \ldots, R_{n}\right\rangle$ with $n$ accessibility relations, and any $w \in F$, the frame $\mathcal{F}(w)^{s}$ strongly generated by $w$ in $\mathcal{F}$ is the set of all $a \in \mathcal{F}$, were $a=w$ or

$$
w Q_{i 1} w_{1}, w_{1} Q_{i 2} w_{2}, \ldots, w_{k-1} Q_{k} w_{k}, w_{k}=a,
$$

for some $Q_{j} \in\left\{R_{1}, \ldots R_{n}, R_{1}^{-1}, \ldots R_{n}^{-1}\right\}, w_{i} \in \mathcal{F}$.

Definition 18. We say a class of frames $\mathcal{K}$ admits an universal formula if there is a formula $\square_{u}(x)$ (called universal formula) constructed from a single letter $x$ and logical operations, such that the following holds. For any $\mathcal{F} \in \mathcal{K}$, any world $w \in \mathcal{F}$ and any valuation $V,(\mathcal{F}, w) \Vdash_{V} \square_{u}(x)$ if and only if $(\mathcal{F}, a) \Vdash_{V} x$, for all $a \in \mathcal{F}(w)^{s}$.

A logic $\mathcal{L}$ admits an universal modality if, for some class of frames $\mathcal{K}$, $\mathcal{L}=\mathcal{L}(\mathcal{K})$ and $\mathcal{K}$ admits an universal formula. The logic $\mathcal{T} \mathcal{L}\left(\mathcal{N}_{C}^{\square_{w}^{+-}}\right)$admits universal modality, the term (formula)

$$
\square_{u}(x):=\neg \diamond^{-} \neg x \wedge \neg \diamond^{+} \neg x
$$


is an universal formula for the class of frames generating $\mathcal{T} \mathcal{L}\left(\mathcal{N}_{C}^{\square_{w}^{+-}}\right)$. Based on this we can prove Lemma 19. Recall that, for a $\operatorname{logic} \mathcal{L}$, a formula $\varphi$ is unifiable (in $\mathcal{L}$ ), if there is a substitution $\sigma$, where $\sigma \varphi \in \mathcal{L}$.

Lemma 19. A rule $\varphi / \psi$ is admissible in $\mathcal{T} \mathcal{L}\left(\mathcal{N}_{C}^{\square_{w}^{+-}}\right)$if and only if $\neg \diamond^{-} \neg \varphi \wedge \neg \diamond^{+} \neg \varphi \rightarrow \psi \in \mathcal{T} \mathcal{L}\left(\mathcal{N}_{C}^{\square_{w}^{+-}}\right)$, or $\varphi$ is not unifiable in $\mathcal{T} \mathcal{L}\left(\mathcal{N}_{C}^{\square_{w}^{+-}}\right)$.

Proof. If $\varphi$ is not unifiable, then evidently $\varphi / \psi$ is admissible. If $\varphi / \psi$ is valid in all frames $\mathcal{N}_{C}$ then we immediately infer that $\varphi / \psi$ is admissible. For the converse, suppose that $\varphi / \psi$ is not valid in some $\mathcal{N}_{C}, \mathcal{L}_{C} \nVdash \varphi / \psi$, but there is a substitution $\sigma$ such that $\sigma \varphi \in \mathcal{T} \mathcal{L}\left(\mathcal{N}_{C}^{\square^{+-}}\right)$. Take the universal formula $\square_{u}(x)$ and the substitution $\varepsilon x_{i}:=\left(\square_{u}(\varphi) \wedge x_{i}\right) \vee\left(\neg \square_{u}(\varphi) \wedge \sigma x_{i}\right)$ for every variable letter $x_{i}$ from $\varphi / \psi$.

Consider an $\mathcal{N}_{C}$, a valuation $V$ of letters from $\varepsilon \varphi$ in $\mathcal{N}_{C}$ and an $a \in \mathcal{N}_{V}$. If $\left(\mathcal{N}_{C}, a\right) \Vdash_{V} \square_{u}(\varphi)$ then, for any $b \in \mathcal{N}_{C}(a)^{s},\left(\mathcal{N}_{C}, b\right) \Vdash_{V} \square_{u}(\varphi)$ and $\left(\mathcal{L}_{C}, b\right) \Vdash_{V} \varphi$. Therefore, for any $b \in \mathcal{N}_{C}(a)^{s},\left(\mathcal{N}_{C}, b\right) \Vdash_{V} \varepsilon x_{i} \equiv x_{i}$ and hence $\left(\mathcal{N}_{C}, a\right) \Vdash_{V} \varepsilon \varphi$.

If $\left(\mathcal{N}_{C}, a\right) \nVdash_{V} \square_{u}(\varphi)$ then, for any $b \in \mathcal{N}_{C}(a)^{s},\left(\mathcal{N}_{C}, b\right) \nVdash_{V} \square_{u}(\varphi)$ and consequently $\left(\mathcal{N}_{C}, b\right) \Vdash_{V} \varepsilon x_{i} \equiv \sigma x_{i}$. Therefore from $\sigma \varphi \in \mathcal{T} \mathcal{L}\left(\mathcal{N}_{C}^{\square_{w}^{+-}}\right)$we conclude for any $b \in \mathcal{N}_{C}(a)^{s},\left(\mathcal{N}_{C}, b\right) \Vdash_{V} \varepsilon \varphi$, in particular, $\left(\mathcal{N}_{C}, a\right) \Vdash_{V} \varepsilon \varphi$. Thus, we proved $\varepsilon \varphi \in \mathcal{T} \mathcal{L}\left(\mathcal{N}_{C}^{\square_{w}^{+-}}\right)$.

From $\mathcal{N}_{C} \nVdash \varphi / \psi$ for some $\mathcal{N}_{C}$, we have, for a valuation $V_{1}, \mathcal{N}_{C} \Vdash_{V_{1}} \varphi$ and $\left(\mathcal{N}_{C}, a\right) \nVdash_{V_{1}} \psi$ for an $a \in \mathcal{N}_{C}$. Then $\mathcal{N}_{C}(a)^{s} \Vdash_{V_{1}} \varphi$, and, for any $b \in \mathcal{N}_{C}(a)^{s}$, $\left(\mathcal{N}_{C}, b\right) \Vdash_{V_{1}} \square_{u}(\varphi)$, and $\left(\mathcal{N}_{C}, b\right) \Vdash_{V_{1}} \varepsilon x_{i} \equiv x_{i}$, so $\left(\mathcal{N}_{C}, a\right) \nVdash_{V_{1}} \varepsilon \psi$, hence $\varepsilon \psi \notin \mathcal{T} \mathcal{L}\left(\mathcal{N}_{C}^{\square_{w}^{+-}}\right)$.

In the light of this lemma, we only need to recognize valid consecutions and unifiable formulas. For a multi-modal logic $\mathcal{L}, \mathcal{L}$ is said to have no irreflexive terminal points if $\neg \square_{i} \perp \in \mathcal{L}$ holds for all modal operations $\square_{i}$.

Lemma 20. If a logic $\mathcal{L}$ has no irreflexive terminal points then unification in $\mathcal{L}$ is recognizable in exponential time (NP-complete).

Proof. This is trivial. Indeed, if $\varepsilon: x_{i} \rightarrow \psi_{i}$ is a unifier for $\varphi$ in $\mathcal{L}$, then for the substitution $\varepsilon_{1}: p_{j} \rightarrow \top$, the composition $\varepsilon_{1} \varepsilon$ is again a unifier for $\varphi$. Since $\neg \square_{i} \perp \in \mathcal{L}, \varepsilon_{1} \psi_{i} \equiv \top \in \mathcal{L}$ or $\varepsilon_{1} \psi_{i} \equiv \perp \in \mathcal{L}$. So, it is sufficient to check as unifiers for $\varphi$ only substitutions in $\{\top, \perp\}$. Computation of values of such substitutions is polynomial, again since $\neg \square_{i} \perp \in \mathcal{L}$. Thus, overall complexity is the same as satisfiability in PC, i.e. the tack is NP-complete. 
This lemma evidently works for our logic $\mathcal{T} \mathcal{L}\left(\mathcal{N}_{C}^{\square_{w}^{+-}}\right)$also. Therefore the Theorem 21 below immediately follows from Theorem 16 and Lemma 19.

TheOrEm 21. The logic $\mathcal{T} \mathcal{L}\left(\mathcal{N}_{C}^{\square^{+-}}\right)$is decidable with respect to admissible inference rules. There is an algorithm recognizing inference rules admissible in $\mathcal{T} \mathcal{L}\left(\mathcal{N}_{C}^{\square_{w}^{+-}}\right)$.

Conclusion. The logic $\mathcal{T} \mathcal{L}\left(\mathcal{N}_{C}^{\square_{w}^{+-}}\right)$is seemed to be rather interesting because it possesses features which are problematic to model in conventional modal or temporal languages. This is because $\mathcal{T} \mathcal{L}\left(\mathcal{N}_{C}^{\square}{ }^{+-}\right)$express the properties of the second order logic. We propose an algorithm for recognizing logical laws of $\mathcal{T} \mathcal{L}\left(\mathcal{N}_{C}^{\square_{w}^{+-}}\right)$(so, we proved that $\mathcal{T} \mathcal{L}\left(\mathcal{N}_{C}^{\square_{w}^{+-}}\right.$) is decidable) but there is a good avenue for future research. Say, it would be interesting to find axiomatization for $\mathcal{T L}\left(\mathcal{N}_{C}^{\square_{w}^{+-}}\right)$. The study of the strong unification problem for $\mathcal{T} \mathcal{L}\left(\mathcal{N}_{C}^{\square_{w}^{+-}}\right)$ is interesting and important, it is an open problem now whether there is an algorithm verifying strong unifiability in $\mathcal{T} \mathcal{L}\left(\mathcal{N}_{C}^{\square_{w}^{+-}}\right)$.

\section{References}

[1] Areces G., P. Blackburn, and M. Marx, "The computational complexity of hybrid temporal logics", Logic Journal of IGPL 8 (2000), 653-679.

[2] Bull, R. A., "An algebraic study of tense logics with linear time", The Journal of Symbolic Logic 33 (1968), 27-38.

[3] Bull, R. A., "Note on a paper in tense logic", The Journal of Symbolic Logic 34 (1969), 215-218.

[4] Chagrov, A., "A decidable logic with undecidable admissibility problem for inference rulres", Algebra and Logic 31 (1992), 53-55.

[5] Gabbay, D. M., I. M. Hodkinson, and M. A. Reynolds, "Temporal logic", Mathematical Foundations and Computational Aspects, vol. 1, Clarendon Press, Oxford, 1994.

[6] Gabbay, D. M., and I. M. Hodkinson, "An axiomatisation of the temporal logic with Until and Since over the real numbers", Journal of Logic and Computation 1 (1990), 229-260.

[7] Ghilardi, S., "Unification in intuitionistic logic", Journal of Symbolic Logic 64, 2 (1999), 859-880.

[8] Ghilardi, S., "Best solving modal equations", Annals of Pure and Applied Logic 102 (2000), 183-198. 
[9] Ghilardi, S., and L. Sacchetti, "Filtering unification and most general unifiers in modal logic", Journal of Symbolic Logic 69, 3 (2004), 879-906.

[10] Goldblatt, R., "Logics of time and computation", CSLI Lecture Notes, no. 7, 1992.

[11] Goldblatt, R., "Mathematical modal logic: A view of its evolution", J. Applied Logic 1, 5-6 (2003), 309-392.

[12] Golovanov, M.I., A.V. Kosheleva, and V.V. Rybakov, "Logic of visibility, perception, and knowledge and admissible inference rules", Logic Journal of the IGPL 13, 2 (2005), 201-209.

[13] Goranko, V., and S. Passy, "Using the universal modality, Gains and questions", Journal of Logic Computation 2, 1 (1992), 5-30.

[14] Harrop, R., "Concerning formulas of the types $A \rightarrow B \vee C, A \rightarrow \exists x B(x)$ in intuitionistic formal system", J. of Symbolic Logic 25 (1960), 27-32.

[15] Iemhoff, R., "On the admissible rules of intuitionistic propositional logic", J. of Symbolic Logic 66 (2001), 281-294.

[16] Jerábek, E., "Admissible rules of modal logics", J. of Logic Computation 15 (2005), 411-431.

[17] Kapron, B. M., "Modal sequents and definability", J. of Symbolic Logic 52 (1987), 3, 756-765.

[18] Hintikka, J., and F. Vandamme, Logic of Discovery and Logic of Discourse, Springer, 1986.

[19] Litak, T., and F. Wolter, "All finitely axiomatizable tense logics of linear time flows are coNP-complete", Studia Logica 81, 2 (2005), 153-165.

[20] Lorenzen, P., Einführung in die operative Logik und Mathematik, BerlinGöttingen, Heidelberg, Springer-Verlag, 1955.

[21] Mints, G.E., "Derivability of admissible rules", J. of Soviet Mathematics 6, 4 (1976), 417-421.

[22] Roziere, P., "Admissible and derivable rules", Math. Structures in Computer Science 3 (1993), 129-136.

[23] Rybakov, V.V., "A criterion for admissibility of rules in the modal system S4 and the intuitionistic logic", Algebra and Logic 23, 5 (1984), 369-384 (Engl. Translation).

[24] Rybakov, V.V., Admissible Logical Inference Rules, series "Studies in Logic and the Foundations of Mathematics", vol. 136, Elsevier Sci. Publ., NorthHolland, 1997. 
[25] Rybakov, V. V., "Construction of an explicit basis for rules admissible in modal system S4", Mathematical Logic Quarterly 47, 4 (2001), 441-451.

[26] Rybakov, V. V., "Logical consecutions in tntransitive temporal linear logic of finite intervals", Journal of Logic Computation 15, 5 (2005), 633-657.

[27] Rybakov, V. V., "Logical consecutions in discrete linear temporal logic", Journal of Symbolic Logic 70, 4 (2005), 1137-1149.

[28] Rybakov, V. V., "Linear temporal logic with Until and Before on integer numbers, deciding algorithms", pp. 322-334 in Computer Science - Theory and Applications, Lecture Notes in Computer Science, Springer, vol. 3967, 2006.

[29] Rybakov, V. V., "Branching time logic $\mathcal{T} \mathcal{L}\left(\mathcal{N}_{C}^{\square_{w}^{+-}}\right)_{\alpha}$ with operations Until and Since based on bundles of integer numbers", Logical Consecutions, Deciding Algorithms, 2006 (submitted).

[30] Segerberg, K., "Modal logics with linear alternative relations", Theoria 36 (1970), 301-322.

[31] van Benthem, J., The Logic of Time, Reidel, Dordrecht, Synthese Library, vol. $156,1983$.

[32] van Benthem, J., and J. A. Bergstra, "Logic of transition systems", Journal of Logic, Language and Information 3, 4 (1994), 247-283.

\author{
V. RYBAKov \\ Department of Computing and Mathematics \\ Manchester Metropolitan University \\ John Dalton Building, Chester Street \\ Manchester M1 5GD, U.K. \\ V. Rybakov@mmu . ac . uk
}

